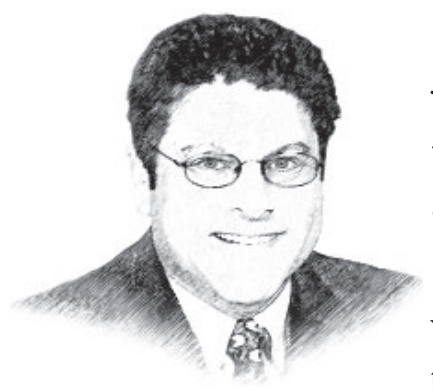

\title{
Potential systemic benefits of shocking or blocking nerves
}

While hormone therapy is demonstrably effective for vasomotor symptoms for the vast majority of perimenopausal and postmenopausal women, for some patients it is not an acceptable choice. In this issue of the Journal, Lee et $\mathrm{al}^{1}$ discuss stellate ganglion block (SGB), the guided percutaneous injection of anesthetic to relieve vasomotor symptoms, as an alternative in women for whom estrogen replacement is not a good option or has failed along with other approaches to provide adequate symptom control.

Studies supporting the efficacy of SGB are fairly small and of short duration. The reported efficacy varies, perhaps indicating a need for patient selection criteria, and perhaps indicating a significant dependence on the skill of the proceduralist. My takeaway is that this is a potential niche therapy for women with few options to control their significant vasomotor symptoms and improve quality of life.

Digging deeper into the topic of nerve manipulation therapies brings to light fascinating heretofore fringe literature on this and other conceptually related approaches of neuromodulation. There are reports of SGB benefitting patients with various upper extremity and facial pain syndromes including migraine. Preclinical research has suggested that nerve fibers connect this sympathetic nerve way station to parts of the brain including the hypothalamus, potentially influencing the impact of hormones on the stress response and the immune system. Isolated reports suggest benefit of SGB in diverse syndromes including posttraumatic stress disorder, dysautonomia, and longhaul COVID-19. If true, the mechanism is likely far more complex than simply blocking regional sympathetic outflow to modulate regional vasoconstriction and dilation. SGB is used to treat other conditions and is featured on the websites of several medical centers.

What has really caught my attention is the expanding research on controlled regional neuromodulation and its impact on systemic physiology and inflammation. Using electrical current to directly affect function of nerve and muscle is well accepted and at least conceptually understandable (eg, electroconvulsive therapy for depression, deep brain stimulation for Parkinson disease, cardiac pacemaker and defibrillator input for heart rhythm control). It is far less readily conceptualized how nerve stimulation can exert effects on systemic inflammation and the immune response.

But fascinating are the studies on stimulating (not blocking as is done with SGB) the vagal nerve, which can be done percutaneously. Several distinct animal models, involving different organs and inflammatory triggers, have been used to demonstrate systemic anti-inflammatory effects stemming from stimulation of the vagal nerve and some of its branches. One hypothesis with some experimental support explains this effect via vagal stimulation of the splenic nerve, which causes acetylcholine release in the spleen. The acetylcholine binds to receptors on macrophages and likely other cells, resulting in downregulation of inflammatory cytokines such as tumor necrosis factor, and also in altered cell circulation. ${ }^{2}$

A recent open-label study ${ }^{3}$ in human patients with rheumatoid arthritis receiving

doi:10.3949/ccjm.89b.03022 
percutaneous vagal nerve stimulation generated promising (though very preliminary) beneficial results using well-accepted clinical, laboratory, and imaging techniques. Larger studies in patients with rheumatoid arthritis are ongoing. A seemingly successful site for percutaneous stimulation is the external ear, where there are branches of the vagal nerve, making this a far more accessible and acceptable approach compared with direct activation of the vagal trunk as used in some animal studies.

While I am not yet ready to accept that the electromagnetic aura of body magnets and copper bracelets may replace methotrexate, it is provocative to wonder whether controlled neuromodulation may in the future provide a useful nonpharmacologic adjunct to the treatment of pain and inflammation. A better understanding of it may even help explain some of the beneficial effects of acupuncture, a procedure that has also in some studies reduced menopausal hot flashes.

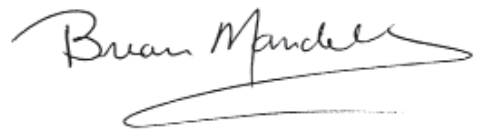

Brian F. Mandell, MD, PhD

Editor in Chief

1. Lee YS, Wie C, Pew S, Kling JM. Stellate ganglion block as a treatment for vasomotor symptoms: clinical application. Cleve Clin J Med 2022: 89(3):147-153. doi:10.3949/ccjm.89a.21032

2. Tynan A, Brines $\mathbf{M}$ and Chavan S. Control of inflammation using non-invasive neuromodulation: past, present, and promise. Int Immunol 2022; 34(2):119-128. doi:10.1093/intimm/dxab073

3. Marsal S, Corominas H, de Augustin JJ, et al. Non-invasive vagus nerve stimulation for rheumatoid arthritis: a proof-of-concept study. Lancet Rheumatol 2021: 3(4)e262-e269. https://doi.org/10.1016/S2665-9913(20)30425-2 